\title{
Affect of Reinforcement Constraints on Shrinkage and Creep Prediction Model of High Performance Concrete
}

\author{
Junli Luo ${ }^{1, a}$, Guanghui Li ${ }^{2, b}$ \\ ${ }^{1}$ Hunan Radio and TV University, Changsha 410075, China \\ ${ }^{2}$ China Machinery International Engineering Design \& Research Institute Co., Ltd., Changsha \\ 410007, China \\ aluojunli@foxmail.com , ’396394027@qq.com
}

\begin{abstract}
Keywords: High performance concrete, Reinforcement constrains, Influence coefficients, Shrinkage and creep, Prediction model

Abstract. Reinforcement constrains of reinforcement applying on concrete mainly depends on three factors: the grade of concrete (elastic modulus), section reinforcement and loading time. The lower grade of concrete, the smaller reinforcement ratio, the longer loading time all make an obvious influence of reinforcement constraints on shrinkage and creep. The simplified calculation formulas of three physical quantities are given respectively and verified in this paper, which considered the above three factors. The three physical quantities are the influence coefficient of reinforcement on creep strain, the influence coefficient of reinforcement on creep curvature, and the influence coefficient of reinforcement on the creep deflection.
\end{abstract}

\section{Introduction}

For Reinforced and Prestressed Concrete, the reinforcement affected significantly on shrinkage and creep of plain concrete, though some standards neglect this effect, such as the European Standard and Chinese Standard. But the impact of reinforcement constrain on shrinkage and creep should not be neglected, which is an inevitable need to perfect the calculation models of shrinkage and creep. Some standards, such as ACI 209R, ACI 318 and Branson's formulas, consider the reinfocement having a combined effect on shrinkage and creep but not considering the effects of reinforcement on shrinkage and creep separately. In addition, the ACI 318 and Branson formulas, overestimate the affects of reinforcement on shrinkage and creep of concrete[1]. The calculated value of Su Qinghong's formula are close to the experimental data frow $\mathrm{Wu} \mathrm{Di}$, but still only the impact of reinforcement ratio is taken into account in these formulas, without consideration of time, concrete grade and eccentricity. H.Trost's formula is more comprehensive considering, such as concrete grade, reinforcement ratio, time, etc., and in a simpler form, but it is only applicable for reinforced concrete members of axial compression. [2,3,4].

Three physical meaning is clearly defined in Hu Di's formula, and the calculation formulas are given out respectely, which is the reinforced influence coefficient on creep strain $k_{s}$, the reinforced influence coefficient on creep curvature $\lambda_{s}$, the reinforced influence coefficient on creep deflection or arch degree $\gamma_{s}[5,6]$. these formulas full considering of comprehensive factors are mainly used in reinforced concrete and prestressed concrete beams, but the form of which is very complex, especially the formula for calculating the degree of curvature and arch. It is not so practicable for engineering. Therefore, it is necessary to establish a set of relatively simple form formulas, which not only the main factors can be fully considered in, but also can be easily calculated.

\section{Constraint influence coefficient of reinforcement applying on creep}

The influence coefficient of reinforcement on creep strain is composed of two parts: The first part is from the constrained impact of reinforcement ratio and steel layout of the concrete cross-section on creep strain. The second part is from the constrained impact of prestressed tendons on creep strain in 
consideration of stress relaxation. By calculated Hu Di's test study, it is found that the second part is much small than the first part. The impact of the second part on the result is less than $2 \%$, and this value will be even smaller if the low relaxation strand in use. Therefore, the second part of the formula (item of prestressed relaxation effects) can be negligible, that is, without considering the effects of prestress relaxation. This is because the prestressed tendons ratio is generally low, and the constraint on creep is also very small. Then you can get that the simplified formula of $k_{s, c r}$ is the same of the influence coefficient on shrinkage strain $k_{s, s h}$.

Reinforced influence coefficient on creep strain( Eq. 1) :

$$
k_{s, c r}\left(t, t_{0}\right)=\frac{1}{1+n \rho \mu\left[1+\chi \cdot \varphi\left(t, t_{0}\right)\right]}=k_{s, s h}\left(t, t_{0}\right) .
$$

Similarly, due to the low reinforcement ratio of prestressed tendons (the impact on the value of $\lambda_{s, c r}$ is less than $2 \%$ ), the stress relaxation of prestressed reinforcement is ignored. Then the formula of reinforced influence coefficient on creep curvature is simplified as the following form( Eq. 2):

$$
\lambda_{s, c r}\left(t, t_{0}\right)=1-\frac{e \varepsilon_{c}\left(t_{0}\right)}{r^{2} \theta_{c}\left(t_{0}\right)} \cdot \frac{n \mu\left[1+\chi \cdot \varphi\left(t, t_{0}\right)\right]}{1+n \rho \mu\left[1+\chi \cdot \varphi\left(t, t_{0}\right)\right]} .
$$

In the above formula, the influence of three main factors are all taken into consideration, which is the concrete grade (elastic modulus), the total reinforcement ratio and the loading time. The lower grade of concrete, the smaller reinforcement ratio, the longer loading time all make an obvious influence of reinforced constraints on shrinkage and creep.

In theory, the curvature increment of each section of the beam is produced by the combined action of creep and shrinkage, but in fact, the shrinkage effect is generally not great enough to cause the deformation of the upper arch (or under deflection) of the beam. For the members subjected to symmetrical load, it can be considered that the arch degree (or deflection) of the beam is proportional to the variation of the sectional curvature. Therefore, for the sake of simplicity, it is considered that the constraint influence of reinforcement on the arch degree (or deflection) of the beam is the same as that of the cross section curvature, shown in Eq.3:

$$
\gamma_{s, c r}\left(t, t_{0}\right)=\lambda_{s, c r}\left(t, t_{0}\right)=1-\frac{e \varepsilon_{c}\left(t_{0}\right)}{r^{2} \theta_{c}\left(t_{0}\right)} \cdot \frac{n \mu\left[1+\chi \cdot \varphi\left(t, t_{0}\right)\right]}{1+n \rho \mu\left[1+\chi \cdot \varphi\left(t, t_{0}\right)\right]} .
$$

For rectangular section beams with prestressed concrete, concrete grade C50, $n=5.6 ; e=1 / 4 h$, $\rho=1.5 ; \chi$ can be valued $0.8 ; \varsigma$ can be valued 0.75 . The initial strain of the section $\varepsilon_{c}\left(t_{0}\right)$ is equal to the multiplication of initial curvature $\theta_{c}\left(t_{0}\right)$ and the distance $y$ from section gravity to the neutral axis. These data can be substituted into the Eq.1 and the Eq.3, the simplified formulas are obtained, as shown in Eq.4 and the Eq.5:

$$
\begin{aligned}
& k_{s, c r}\left(t, t_{0}\right)=\frac{1}{1+10 \mu\left[1+0.8 \varphi\left(t, t_{0}\right)\right]}, \\
& \lambda_{s, c r}\left(t, t_{0}\right)=1-\frac{4.2 \mu\left[1+0.8 \varphi\left(t, t_{0}\right)\right]}{1+10 \mu\left[1+0.8 \varphi\left(t, t_{0}\right)\right]}=\gamma_{s, c r}\left(t, t_{0}\right) .
\end{aligned}
$$

where, $\varphi\left(t, t_{0}\right)$ is creep coefficient of plain concrete at loading time $t$. For ordinary concrete it can be approximate valued as follow: for loading time more than 1 month, the creep coefficient may equals to 0.5 ; for more than 3 months it can valued 1.0; for more than 6 months it takes 1.2 ; for more than 1 year valued 1.4; for more than 3 years valued 1.8; when the holding time tends to infinity $t \rightarrow \infty$, the creep 
coefficient can valued $2, \varphi\left(\infty, t_{0}\right)=2$. For high grade concrete, the final creep values should be appropriately reduced.

\section{Constraint influence coefficient of reinforcement applying on shrinkage}

Reinforced influence coefficient on shrinkage strain:

$$
k_{s, s h}\left(t, t_{0}\right)=\frac{1}{1+n \rho \mu\left[1+\chi \cdot \varphi\left(t, t_{0}\right)\right]} .
$$

For prestressed concrete beams with rectangular cross section, Eq. 6 can be simplified as follow:

$$
k_{s, s h}\left(t, t_{0}\right)=\frac{1}{1+10 \mu\left[1+0.8 \varphi\left(t, t_{0}\right)\right]} .
$$

For non prestressed and partially prestressed concrete members, the shrinkage effect may cause an overloaded tensile stress in some local, which can make cracks in the beam body. This means the concrete shrinkage have great influence on the stress of concrete. While, the effect of concrete shrinkage affected on deflection is mainly shown as the deformation along the length direction of the beam body, but not great enough to cause the upper arch or lower deflection of the beam. However, due to the constraint of beam end bearing and the temperature effect, the shrinkage deformation of concrete caused by concrete shrinkage is also very limited. In order to simplify the calculation, the influence of the reinforcement on the concrete shrinkage (arch degree or deflection) can be negligible, and the influence of reinforcement on the shrinkage curvature of cross section is also negligible. So we can get Eq. 8:

$$
\lambda_{s, s h}=\gamma_{s, s h}=1
$$

\section{Verification of the constraint influence coefficient of reinforcement}

The curves in Fig. 1 are the value of influence coefficient of reinfocement on the creep coefficient, according to $\mathrm{Hu}$ Di's experiment and calculation. In the calculation example, the prestressed concrete beams is of rectangular section, and the concrete grede is C50. The other parameters valued as follows: for ordinary steel strand $\alpha_{\sigma}=5$; prestressed reinforcement ratio $\mu_{p}=0.28 \%$; the ordinary reinforcement ratio $\mu_{s}=0.85 \%$; For C50 concrete, we can obtain that $n=6 ; \chi=0.82 ; \varsigma=0.75$.

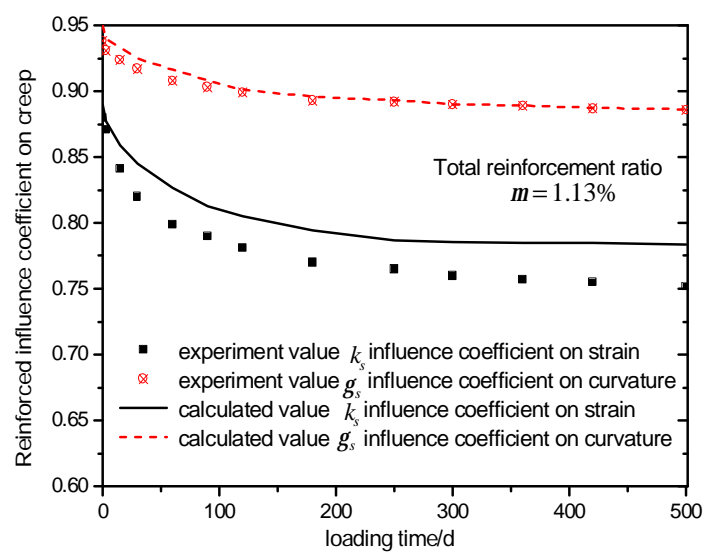

Fig.1 Reinforced influence coefficient on creep developed with time

The scattered points in Fig. 1 is the calculated values of influence coefficient reinforcement on creep, according to the Eq.5 and Eq.4. The loading time for calculation is $3 \mathrm{~d}, 30 \mathrm{~d}, 90 \mathrm{~d}, 180 \mathrm{~d}, 360 \mathrm{~d}, 500 \mathrm{~d}$, respectively. It can be seen that the calculated values are very close to $\mathrm{Hu}$ Di's experimental values, and the maximum error is only $3.5 \%$. It is said that the simple form calculation formula has good accuracy . 
Zhang Yuntao and Pan Zuanfeng carried out a test of shrinkage and creep of C60 reinforced concrete. The influence of different reinforcement ratio $(0.38 \%, 0.76 \%, 1.71 \%)$ on the shrinkage strain and creep strain is obtained. Because it is an axial compression test, the eccentricity distance $e=0$, that is $\rho=1$. By comparison of the calculatd influence coefficient (by Eq.2) and the Zhang Yuntao's test value, it can be seen that: the calculation values are in good agreement with the experimental data, and can satisfy the engineering demand, shown in Fig.2.

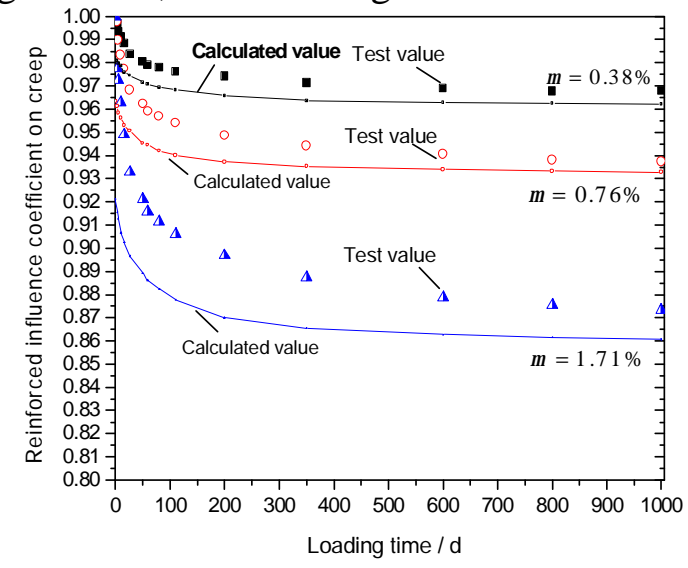

Fig.2 Reinforced influence coefficient on creep

\section{Conclusions}

Reinforcement constrains of reinforcement applying on concrete mainly depends on three factors: the grade of concrete (elastic modulus), section reinforcement and loading time. The simplified calculation formulas of three physical quantities are given respectively and verified in this paper, which considered the above three factors. The three physical quantities are the influence coefficient of reinforcement on creep strain, the influence coefficient of reinforcement on creep curvature, and the influence coefficient of reinforcement on the creep deflection.

\section{References}

[1] Luo J L, Xu Z S, Zhao D: Review of Long-Term Deformation of Recycled Coarse and Fine Aggregate Concrete. Applied Mechanics and Materials, 2014, 448:734-737.

[2] Hu Di, Chen Zhengqing: Experimental research on the deformations for shrinkage and creep of beams in prestressed concrete bridge. China Civil Engineering Journal, Vol.36(8)(2003), p.80

[3] Luo J L, Xu Z S, Li J, et al : Study on Prediction Model of Shrinkage and Creep of Prestressed Concrete Beam in Passenger Dedicated Line[J]. Advanced Materials Research, 2011,255:3998-4002. [4] Luo J L, Xu Z S, Shen Y J : Review on the optimization approach of the long term deformation performance of recycled concrete. Journal of Safety and Environment, 2013,13(05):212-217. [5] Luo J L, Xu Z S, Xie B C: Shrinkage and Creep properties of different grade recyckled aggregate concretes. Jouranl of Central South University(Science and Technology),2013,44(09):3815-3822.

[6] Luo J L, Xu Z S, Xiong W: Effect of Recycled Aggregate on shrinkage and creep of HPC: Comparative Trial. Industrial Construction, 2014(01):79-83. 